\title{
Estudio de pacientes transeúntes en una unidad de hemodiálisis
}

\author{
Lerma García, Daniel \\ Sausa Micó, Verónica \\ Linarejos Huertas, Belén
}

Servicio de Nefrología.

Unidad de Hemodiálisis.

Hospital Can Misses. Ibiza.

\begin{abstract}
RESUMEN
Por la situación de nuestra unidad en un destino turístico importante, son muchos los pacientes que nos demandan tratamiento. Los enfermeros nos encontramos con retos añadidos al carácter ambulatorio del paciente como son el idioma, el diferente manejo terapéutico de los pacientes en diálisis de otros países y la falta de registros enfermeros que nos permitan poder desarrollar planes de cuidados que garanticen una continuidad en el plan terapéutico.
\end{abstract}

El objetivo del estudio es caracterizar el tipo de pacientes transeúntes que recibieron tratamiento dialítico en nuestra unidad de hemodiálisis durante los meses de mayo-octubre en los años 2001 y 2002. Se ha realizado un estudio observacional, descriptivo y retrospectivo de una muestra total de 17 pacientes. Para la recogida de datos, se ha utilizado un registro de elaboración propia dividida en tres apartados: datos personales, pauta de diálisis y complicaciones intradiálisis. Un elemento que se hace necesario para garantizar la continuidad de los cuidados de enfermería es el registro para los pacientes transeúntes que debería acompañar a cada uno en sus desplazamientos a otras unidades de diálisis.

PALABRAS CLAVE: PACIENTES TRANSEÚNTES

CUIDADOS

HEMODIÁLISIS

NEFROLOGÍA

\section{STUDY OF OUTPATIENTS IN A HAEMODIALYSIS UNIT}

\section{ABSTRACT}

Due to the location of our unit in an important tourist destination, there are many patients who ask us for treatment. Nursing staff face challenges in addition to the clinical nature of the patient, such as language, the different therapeutic handling of patients in dialysis in other countries and the lack of nursing registers to help us develop care plans to guarantee continuity in the therapeutic plain.

The aim of the study is to characterise the type of outpatients who received treatment in our haemodialysis unit from May to October in the years 2001 and 2002. An observational, descriptive and retrospective study was made of a total sample of $17 \mathrm{pa}-$ tients. One of our own registers was used in the data collection, divided into three sections: personal data, dialysis tendency and intradialysis complications. One element that is necessary to guarantee the continuity of the nursing care is the outpatients register, which must accompany each person when they travel to other dialysis units.

KEYWORDS: OUTPATIENTS

CARE

HAEMODIALYSIS

NEPHROLOGY 
La Insuficiencia Renal Crónica Terminal (IRCT) es una enfermedad que conduce inevitablemente a la necesidad de una terapia sustitutiva de la función renal perdida. El tratamiento de elección en la actualidad es el trasplante renal, pero mientras éste se hace posible, es necesario someter al paciente a técnicas de depuración sanguínea ${ }^{(1,2)}$.

Una vez evaluado al paciente y de acuerdo con él, se le incluye en programas de diálisis peritoneal o de hemodiálisis. Si bien el auge de la diálisis peritoneal continúa ambulatoria sigue experimentando un incremento, es la hemodiálisis la técnica más utilizada en la sustitución de la función renal ${ }^{(3,4)}$. Tras la inclusión en el programa de diálisis, todos los esfuerzos del personal sanitario que los atiende se centran en proporcionar a los pacientes estrategias para aumentar su calidad de vida y la autonomía necesaria para mantener un estilo de vida lo más normalizado posible ${ }^{(5,6)}$.

Los pacientes pueden sentirse limitados en diferentes ámbitos de su vida social y personal, para lo que es necesario el apoyo de un equipo multidisciplinar que le permita aprender a desarrollar estrategias para vencer estos obstáculos ${ }^{(7-9)}$. Uno de los problemas con los que se pueden encontrar los pacientes renales incluidos en programas de hemodiálisis es la dificultad para viajar, pues necesitan tener acceso a una unidad de diálisis que les ofrezca la posibilidad de recibir tratamiento sustitutivo durante el periodo de tiempo deseado(10,11). Actualmente, los trámites necesarios para realizar hemodiálisis ambulatorias en otros centros los gestiona, bien el propio paciente, el equipo de nefrología o asociaciones de enfermos renales.

Por la situación de nuestra unidad en un destino turístico importante durante el periodo vacacional, son muchos los pacientes que demandan tratamiento durante espacios de tiempo más o menos largos. Debido a esta excesiva demanda, no siempre es posible poder recibir en nuestra unidad a todos los pacientes que eligen como destino veraniego la isla. Esta situación viene marcada sobre todo por el escaso número de plazas de que disponemos para ofertar a pacientes transeúntes.

Por otra parte, los enfermeros nos encontramos además, con otros retos añadidos al carácter ambulatorio del paciente como son el idioma, el diferente manejo terapéutico de los pacientes de otros países y la falta de registros enfermeros que nos permitan poder desarrollar planes de cuidados que garanticen una continuidad en el plan terapéutico(12,13).

Aun así, en un periodo de doce meses (seis en 2001 y seis en 2002) hemos recibido en nuestra unidad un nú- mero total de 17 pacientes para diálisis ambulatoria, que supone un número elevado, dada la escasez de plazas disponibles.

\section{OBJETIVOS}

\section{Objetivo principal}

- Caracterizar el tipo de pacientes transeúntes que recibieron tratamiento dialítico en nuestra unidad de hemodiálisis durante los meses de mayo-octubre en los años 2001 y 2002.

\section{Objetivos específicos}

- Valorar la tolerancia dialítica de los pacientes transeúntes en nuestra unidad de hemodiálisis.

- Valorar la pauta de diálisis más significativa de los pacientes que realizan hemodiálisis eventuales en nuestra unidad.

- Determinar la adecuación de la dosis de diálisis periódica de los pacientes dializados en nuestra unidad.

- Determinar la procedencia más habitual de los pacientes que reciben el tratamiento en nuestra unidad.

\section{MATERIAL Y MÉTODOS}

\section{Caracterización de los pacientes}

Los datos recogidos pertenecen al total de pacientes transeúntes recibidos en la Unidad de Hemodiálisis del Hospital Can Misses de Ibiza, en el periodo comprendido entre los meses de mayo a octubre de los años 2001 y 2002. Se ha realizado un estudio observacional, descriptivo y retrospectivo de una muestra total de 17 pacientes con un rango de edad entre los 25 y los 76 años, que supone una media de edad de 53,94 años y una desviación típica de $\pm 15,99$.

Respecto al sexo, el $64,70 \%$ son varones y el $35,30 \%$ son mujeres. El 41,17\% recibieron tratamiento en el 2001 y el 58,83\% lo recibieron en el 2002. De todos los pacientes que recibieron tratamiento dialítico, el $76,5 \%$ procedían de diferentes ciudades españolas, repartiéndose de la siguiente forma: el 38,46\% de Barcelona, el 15,38\% de Madrid, 7.69\% de Mallorca, el 7,69\% de Sevilla, el 7,69\% de Murcia, el 7,69\% de Badajoz, el 7,69\% de Alicante y el 7,69\% de Valencia (figura 1). De los pacientes procedentes del extranjero (el 23,5\%), el 50\% eran de nacionalidad alemana, el 25\% marroquí y el 25\% restante, irlandesa.

El intervalo de tiempo de estancia en la isla recibiendo tratamiento se reparte de la siguiente manera: el 17,64\% un día, el 35,29\% una semana, el 11,76\% dos semanas, 
el $29,41 \%$ un mes y el $5,88 \%$ dos meses. El tiempo de diálisis total para estos pacientes supone 21.420 minutos (357 horas) de tratamiento.

La enfermedad primaria causante de la IRCT es, en el $47,05 \%$ de los casos, una nefropatía no filiada; en el $23,52 \%$, una nefropatía intersticial; en el 11,76\%, una enfermedad poliquísitica; en el 11,76\%, una glomerulonefritis y en el 5,88\%, nefropatía por analgésicos.

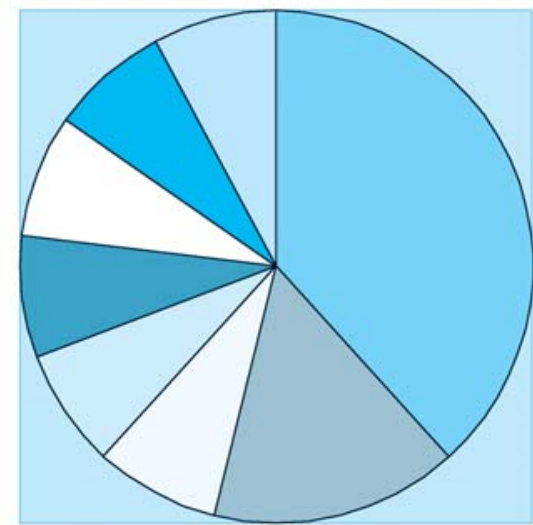

\section{$\square$ Barcelona \\ Madrid \\ Mallorca \\ $\square$ Sevilla \\ Murcia \\ $\square$ Badajoz \\ Alicante \\ $\square$ Valencia}

Figura 1. Lugares de procedencia de los pacientes.

El tipo de acceso vascular que portan los pacientes es FAVI radial derecha el 29,41\%, el 23,52\% FAVI radial izquierda, el 11,76\% FAVI humeral derecha, el 11,76\% goretex humeral derecho, el 5,88\% FAVI humeral izquierda, el 5,88\% catéter subclavio derecho, el 5,88\% catéter femoral derecho y el 5,88\% catéter yugular derecho (figura 2).

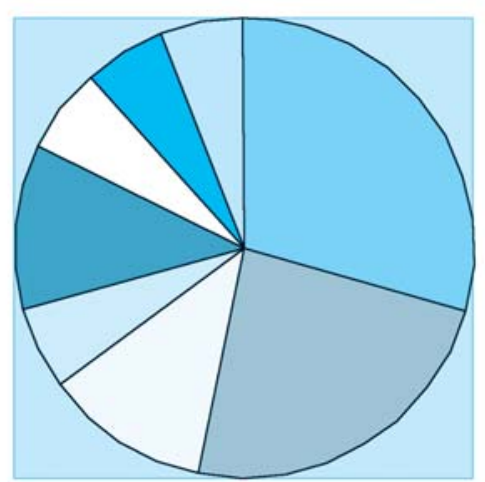

$\square$ FAVI radial
derecha
$\square$ FAVI radial
izquierda
$\square$ FAVI humeral
derecha
$\square$ FAVI humeral
izquierda
$\square$ goretex humeral
derecho
$\square$ catéter subclavio
derecho
$\square$ catéter femoral
derecho
$\square$ catéter yugular
derecho

Figura 2. Tipo de accesos vasculares.

\section{Metodologia}

Para la recogida de datos, se ha utilizado un registro de elaboración propia dividido en tres apartados: datos personales, pauta de diálisis y complicaciones intradiálisis.
En el primer apartado, se incluye el registro de la edad, el sexo, mes y año en que recibió tratamiento en la unidad, procedencia del paciente, horas de diálisis totales en la unidad, enfermedad primaria causante de la IRCT y los valores del KTV y del PCR.

En la segunda parte, se recoge el tiempo de cada sesión, el tipo de acceso vascular, la técnica de punción, el flujo de bomba arterial, la técnica de diálisis, el tipo de dializador, la pauta de heparinización, el calcio del baño, la conductividad y la medicación administrada. También recogemos si en el informe para la hemodiálisis eventual se incluye un informe de enfermería que acompañe al informe médico ${ }^{(14,15)}$.

La última parte del registro contempla las diferentes complicaciones dialíticas y la actuación de enfermería ante ellas.

\section{RESULTADOS}

En cuanto a los resultados, el 70,58\% de los pacientes demandaron diálisis de 240 minutos, el 17,63\% de 210 minutos y el $11,76 \%$ de 180 minutos. Como técnica de punción se utilizó en el 92,85\% la bipunción y en el $7,15 \%$ tuvo que ser utilizada la unipunción. El flujo medio de bomba durante la sesión fue de 298,23 ml/min con una técnica de diálisis convencional en el 94,11\% y con perfiles de peso y conductividad en el $5,89 \%$ de los casos.

El tipo de dializador empleado en el $41,17 \%$ fue tanto el Diacepal 16 como el P200. El hemodializador NT 1665 se usó en el 11,76\% y el Crystal 2800 en el $5,88 \%$ de las diálisis. El 23,52\% de los pacientes aportaba registro de enfermería para transeúntes en el informe para hemodiálisis eventuales.

La conductividad utilizada durante las sesiones fue 14 en el $70,58 \%$ y de 14,5 en el $29,42 \%$. En cuanto al calcio del baño, en el 88,23\% fue de 2,5 y en el 11,77 de 3,5.

En referencia a la heparinización, en el 47,05\% se utilizó bolo inicial (20, 30 ó 40 mg) y heparina contínua (10 $\mathrm{mg} / \mathrm{h}$ ). El 41,17\%, realizó la sesión con heparina de bajo peso molecular (Clexane de $20 \mathrm{mg}$ y $40 \mathrm{mg}$ ), mientras que el $11,76 \%$ sólo necesitó heparina en bolo inicial.

Como medicación, el 52,94\% de los pacientes recibió alguna dosis de eritropoyetina, el 29,41\% aportes de hierro intravenoso y el $5,88 \%$ calcitriol intravenoso postdiálisis. El 47,05\% no recibió ningún tipo de tratamiento farmacológico. 
En cuanto a las complicaciones agudas intradiálisis, el $17,64 \%$ sufrió calambres e hipotensión; el 11,76\% calambres de diferente intensidad y el 5,88\% hipotensión. El $64,70 \%$ no sufrió ningún tipo de complicación aguda (figura 3).

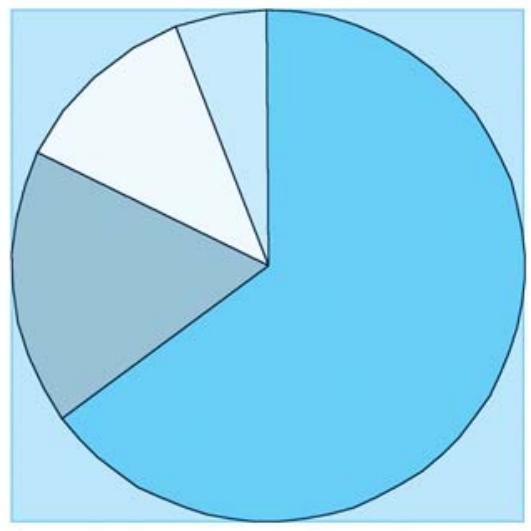

\section{Ninguna}

$\square$ Calambres e hipotensión

Calambres

Hipotensión

Figura 3. Complicaciones agudas de los pacientes.

\section{DISCUSIÓN}

El cuidado de pacientes renales en régimen ambulatorio requiere de una gran especialización en los profesionales que los atienden debido a que el conocimiento previo del paciente no existe y es difícil reconocer en que fase de vivencia de la enfermedad crónica se encuentra.

Cuando el paciente además, procede de otro país diferente, a esta situación se une la diferente percepción de los síntomas que viene marcado por rasgos culturales del lugar a donde pertenece. También es frecuente encontrar pautas de diálisis diferentes en cuanto a tiempo por sesión, sesiones por semana, establecimiento de pesos secos, etc, que no son siempre compatibles con la organización de las unidades de diálisis de destino.

Un elemento que se hace necesario para garantizar la continuidad de los cuidados de enfermería es el registro de enfermería para los pacientes transeúntes que debería acompañar a cada uno en sus desplazamientos a otras unidades de diálisis. Este registro puede ser una herramienta eficaz y beneficioso tanto para el paciente como para la enfermera que debe planificar cuidados de calidad y prevenir complicaciones agudas que comprometan la seguridad del paciente. Aún así, estos son pacientes que suelen estar bien controlados y estabilizados en sus respectivas unidades de referencia, como demuestra la baja incidencia de complicaciones agudas en los individuos estudiados.
Todas estas situaciones suponen inevitablemente, una carga extra de trabajo que debemos asumir los enfermeros de las unidades que recibimos una gran cantidad de pacientes transeúntes en el periodo vacacional.

\section{BIBLIOGRAFÍA}

1. Andreu L, Force E. Vivir con insuficiencia renal. Rev ROL Enf 1998; 328: 105-110.

2. Hernández J.A, Pancorbo P. Enfermo renal y enfermería. Inquietudes 1995; 2: 26-32.

3. Levine $\mathrm{D}$. Cuidados del paciente renal. México: Interamericana-Mc Graw Hill; 1993.

4. Daugirdas J, Ing T. Manual de diálisis. Barcelona: Masson; 1996.

5. VII Seminario Español de la Sociedad Europea de Enfermería de Diálisis y Trasplante. Nuevo perfil del paciente renal: un reto para los profesionales. Valencia: EDTNA/ERCA; 1997.

6. Lerma D, Arrazola M, Garrido J.M. La xerosis en los pacientes en tratamiento con hemodiálisis. Enferm Cientif 2001; 232-233: 73-77.

7. Luis M.T. Diagnósticos enfermeros. Un instrumento para la práctica asistencial. Madrid: Harcourt Brace; 1998.

8. Andreu L, Force E. 500 cuestiones que plantea el cuidado del paciente renal. Barcelona: Masson; 1997.

9. Lerma D, Arnau M.A. Los cuidados de la piel en los pacientes en hemodiálisis. Enferm Nefrol 2000; 12: 6-11.

10. Sellarés V. Manual de nefrología clínica, diálisis y trasplante renal. Madrid: Harcourt Brace; 1998.

11. Lerma D, Arrazola M. Cuidados de Enfermería a pacientes en hemodiálisis. Prerspectiva desde atención primaria. Enferm Científic 2002; 242-243: 63-68.

12. Caravaca M, Jiménez J. Problemática psicosocial de los enfermos renales: hacia una asistencia integral. Biseden 1998; Murcia.

13. Lerma D, Arrazola, M. Efectividad de la hemodiálisis en un paciente con insuficiencia renal crónica terminal. Enferm Nefrol 2002; 19: 50-54.

14. Carpenito J.L. Planes de cuidado y documentación en enfermería. Madrid: Interamericana; 1994.

15. Lerma D. Generalidades y Nefrología Clínica. Cuidados en hemodiálisis. Diálisis peritoneal y pediátrica. Atención integral al paciente renal. Trasplante renal. Jaén: LOGOSS; 2003. 